\title{
Differences mathematics and music teaching-learning process between A Coruna and Lisbon
}

Rocio Chao ${ }^{\text {* }}$,

M Dorinda Mato Vazquez ${ }^{b}$,

Aurelio Chao',

Suggested Citation:

New Trends and Issues Proceedings on Humanities and Social Sciences.

Abstract 
New Trends and Issues Proceedings on Humanities and Social Sciences.

1. Background 
New Trends and Issues Proceedings on Humanities and Social Sciences.

2. Object of Research

3. Methodology

4. Shows

5. Results 
New Trends and Issues Proceedings on Humanities and Social Sciences. 


\section{Conclusions}




\section{References}

Revista Aula de Infantil

Formacion de docentes y educadores en educacion infantil. una apuesta clave para el desarrollo integral de la primera infancia

and Behavioral Sciences, .

Procedia. Social

Aprender a Aprender en Educacion Infantil.

Investigacion

en Educacion Matematica. Comunicaciones de los grupos de investigacion. XV Simposio de la SEIEM

Music and mathematics. ¿From Pythagoras to Fractals?

Teoría de la Educacion. Revista Interuniversitaria, 24

Musica y matematicas: conexiones curriculares para un mayor éxito educativo.

Matematicas y musica en ninos pequenos.

_Matematicas y musica en ninos pequenos.

Tu cerebro y la musica: El estudio científico de una obsesion humana

Musica y Matematicas: la armonía de los numeros.

La afectividad hacia las matematicas.

Revista de Investigacion en Educacion, 